\title{
PHYSICAL ASPECTS OF QUASI-LOCAL BLACK HOLE HORIZONS
}

\author{
ALEX B. NIELSEN \\ Max-Planck-Institut für Gravitationsphysik, \\ Am Mühlenberg 1, D-14476 Golm, Germany \\ alex.nielsen@aei.mpg.de
}

Received 27 July 2011

\begin{abstract}
We discuss some of the physical aspects expected to be associated with black holes. These include Hawking radiation, horizon entropy and cosmic censorship. In particular we focus on whether these properties are more naturally associated to causally defined horizons or quasi-local horizons.
\end{abstract}

Keywords: Black holes; causal horizons; quasi-local horizons.

\section{Introduction}

There are a number of physical effects expected to be associated with black holes. Black holes are now a standard paradigm for explaining a large number of astrophysical phenomena such as quasars, gamma-ray bursts and X-ray binaries. ${ }^{1}$ Such astrophysical black holes are expected to be very simple, described to a good approximation by the Kerr stationary vacuum solution. Much smaller black holes may also be produced in high energy particle collisions, in cosmic rays ${ }^{2}$ or in particle colliders. ${ }^{3}$

From a theoretical viewpoint black holes have provided the background for a great deal of speculation about extensions to the known laws of physics. First, they are expected to contain singularities, or at least regions where the usual description of gravity in terms of general relativity breaks down. Second, the similarities between the classical laws of thermodynamics and the laws of black hole mechanics have led to the conjecture that black holes carry a type of entropy related to their surface area. This idea has motivated the search for the microscopic gravitational degrees of freedom that can explain this entropy through a statistical mechanical state counting argument. Third, black holes are expected to be unstable due to the emission of Hawking radiation. This Hawking radiation should transport energy away from the black hole and lead to the mass of the black hole diminishing. This effect may even lead to the black hole disappearing entirely and this poses a challenge to the accepted description of the universe in terms of unitary quantum evolution. 
It is the hope of many researchers to use the properties of black holes to provide clues about the features a quantum theory of gravity would exhibit. The properties of black holes mentioned above have already led to a number of novel physics concepts that are at least in part inspired by their study. We will examine here a number of ideas that are particularly relevant to the discussion of black hole horizons. Several of the ideas are closely related and connected themes run through them. Several of these ideas have been extended to other types of horizons such as cosmological horizons or the Rindler horizons of accelerated observers, with varying degrees of success, but here we will focus only on those associated with gravitational collapse and the formation of black holes.

A key question in all of this and a question that is also a central theme in the contributions of José Luis Jaramillo ${ }^{4}$ and José Senovilla, ${ }^{5}$ is the question of which type of horizon, if any, should these properties be applied to. There are a great many different types of horizons discussed in the literature. These can be classified into two main approaches to defining the horizon; the global causal event horizon and the quasi-local geometrical horizon. Precise definitions of these concepts can be found in the contributions of José Luis Jaramillo and José Senovilla.

Another issue relevant in the context of gravity is to what extent the various concepts rely on the validity of the Einstein equations. The Einstein equations may receive corrections from a number of sources including compactifications of higherdimensions, supersymmetry or quantum loop corrections. In Einstein gravity there is a direct equivalence between the term $R_{a b} l^{a} l^{b}$ appearing in the Raychaudhuri equation and the null energy term $T_{a b} l^{a} l^{b}$. In Einstein gravity in addition, the horizon-entropy is given by $S=A / 4$ but this is not so in more general theories. Most quasi-local horizon definitions are based on the change of area in null directions. We will see that this may have to be changed to accommodate more general gravitational theories.

Perhaps one of the first to suggest black hole thermodynamics be applied to quasi-local horizons such as apparent horizons rather than event horizons was Hájíček ${ }^{6}$ who conjectured that Hawking radiation originates from the region close to the apparent horizon independently of whether an event horizon exists or not. This idea was further examined by Hiscock ${ }^{7}$ who proposed identifying the entropy with one quarter the area of the apparent horizon and Collins ${ }^{8}$ who obtained a $T d S=d Q$ like relation for apparent horizons. An important contribution was made by Hayward ${ }^{9}$ who defined an outer condition for apparent horizons and was able to show that the area of a future outer trapping horizon is nondecreasing if the null energy condition is satisfied locally on the horizon. These ideas received further attention when it was shown that the microstates of black hole entropy can be counted in loop quantum gravity for a constant area isolated horizon ${ }^{10}$ and in analogue models where a true event horizon is not necessary for the production of Hawking radiation. ${ }^{11}$

Part of black hole physics is founded on rigorously proved mathematical theorems, such as the Penrose singularity theorem or the Hawking area theorem. 
Part of the work is founded on less rigorously formulated conjectures such as the generalized second law or holography and much is founded on analogy, such as black hole thermodynamics. Perhaps the central "miracle" of black hole thermodynamics, the result that gives most credence to the thermodynamics analogy, is the fact that the temperature arising in the laws of black hole mechanics turns out to be exactly the same temperature as the thermal spectrum computed for Hawking radiation. Without this agreement much of the foundation of further results and ideas would be weakened if not entirely removed. A number of standard textbook results are derived for Killing horizons in asymptotically flat spacetimes and part of the original impetus for studying quasi-local horizons was to extend some of these results to more general situations, without the need to assume a global Killing vector field or a certain structure at infinity. Thus the isolated horizons of Ashtekar et $a l .{ }^{12}$ need not be Killing horizons, even though they are constant area null horizons and thus still causal horizons in the narrower sense.

But the problem goes deeper than this. Many of the conjectured extensions to the laws of physics are fundamentally rooted in the question of what it means to be a horizon and are intricately tied up with the fundamental features of these horizons. Do the properties refer to the causal structure of the spacetime or to the geometry? Are the properties local and measurable or nonlocal and idealized?

One of the reasons given originally for preferring event horizons over apparent horizons and the related trapping horizons is the foliation dependence of trapping horizons. Examples of collapse spacetimes are known for which an infinite number of intersecting trapping horizons are found, each at different locations, ${ }^{13}$ whereas the location of the event horizon is known to be foliation independent and unique. This problem forms part of the contributions by José Senovilla. ${ }^{5}$ If physical properties are to be associated with quasi-local geometrical horizons, to what extent do the physical properties of black holes require a resolution to this uniqueness problem?

Another reason for favoring the event horizon over apparent horizons is that for asymptotically flat spacetimes that are asymptotically predictable and satisfy the null energy condition it is known that the apparent horizon should lie behind the event horizon. Therefore whatever physical processes are associated with the apparent horizon would be forever concealed from asymptotic observers. However, there does not appear to be any physical reason to expect the effective null energy condition to hold forever in the future of a black hole. In this case the apparent horizon is expected to appear outside the event horizon. ${ }^{14}$ Nevertheless, there have been statements in the literature that future outer trapping horizons must be spacelike or null. ${ }^{15}$ A counterexample involving quantum fields would not be terribly surprising, although an explicitly worked example would be very interesting.

\section{The Motivating Physical Properties}

The use of horizons and related concepts span a great number of fields in theoretical physics from mathematical and geometrical relativity to quantum gravity through 
numerical relativity and analogue gravity models. We will look at a number of these below, by no means an exhaustive list. Many of the concepts presented below are related and inspired by one another.

Two celebrated results form the basis for most of this work, the Penrose singularity theorem and the Hawking area theorem.

\subsection{Penrose singularity theorem}

For spacetimes satisfying the weak energy condition and admitting a noncompact Cauchy surface, if the spacetime contains a trapped surface then there will exist inextendible (ending at a singularity) null geodesics and the spacetime will be null incomplete. ${ }^{16}$ This theorem effectively states that Einstein gravity predicts its own downfall. If a trapped surface exists somewhere a singularity will form and general relativity will break down. Most researchers expect that general relativity will break down before the formation of a singularity due to quantum gravity effects but there is still no completely understood theory of how this will happen. The trapped surfaces that are used in the proof of the theorem are quasi-local. It is only through the unproved cosmic censorship conjecture that singularities are related to event horizons.

\subsection{Hawking area theorem}

The area of the event horizon is nondecreasing in spacetimes that satisfy the weak energy condition and for which no singularities are visible from infinity. ${ }^{17}$ This growth of the event horizon includes the merger of multiple black holes. The result, together with speculation about the verifiability of the second law of thermodynamics, led Bekenstein to postulate that the area of a black hole is a measure of its entropy. ${ }^{18}$ This in turn led to the formulation of the laws of black hole mechanics in an analogous form to the classical laws of thermodynamics. ${ }^{19}$ We turn now to these laws, paying particular attention to their formulation for quasi-local horizons. Further details are available elsewhere. ${ }^{12,20-22}$

\subsection{Zeroth law}

The zeroth law states that the surface gravity is constant over a horizon in equilibrium. At its most basic the law just requires a function that is constant over a stationary horizon. The zeroth law can either define this function, or define what is meant by equilibrium. Any two functions that are constant over the horizon and thus satisfy a zeroth law can be combined together to give a third function that satisfies the zeroth law.

It is relatively easy to prove a zeroth law of black hole mechanics once one has a definition of surface gravity. But there are different definitions of surface 
gravity. ${ }^{23,24}$ For a static, spherically symmetric metric of the form

$$
d s^{2}=-e^{-2 \phi(r)} \triangle r d t^{2}+\frac{d r^{2}}{\triangle(r)}+r^{2} d \Omega^{2},
$$

the surface gravity given by the usual Killing vector method is

$$
\kappa_{\text {Kil }}=e^{-\phi} \frac{\triangle^{\prime}}{2},
$$

where all functions are evaluated on the horizon. The surface gravity defined in terms of the Kodama vector ${ }^{20}$ is

$$
\kappa_{\mathrm{Kod}}=\frac{\triangle^{\prime}}{2},
$$

again with all functions evaluated on the horizon. If the value of the metric function $\phi$ at the horizon is not equal to zero these two definitions will not be equal, although both will satisfy a zeroth law. There are many static black hole solutions for which $\phi$ cannot be set to zero at the horizon. ${ }^{25}$ For the definitions given for isolated horizons $^{12}$ there are in fact an infinite number of different surface gravities all of which satisfy the zeroth law.

\subsection{First law}

There are various interpretations of the first law. In ordinary thermodynamics the first law relates the change in the internal energy to the heat flow and work done. If energy is to appear in an equivalent black hole version of the first law then we must face the problem of defining energy in general relativity, a notoriously difficult problem in itself. In the textbook definitions this problem is solved either by using the asymptotic spacetime mass, the ADM mass, ${ }^{19}$ or using an intergated horizon flux and defining something resembling an integrated Bondi flux at the horizon. ${ }^{26}$

For the static spherically symmetric Reissner-Nordström solution we have that the areal radius of both the event horizon and trapping horizon is given by $r_{H}=$ $M+\sqrt{M^{2}-Q^{2}}$ where $M$ is the ADM mass and $Q$ the asymptotically measured charge. Thus the area difference between two Reissner-Nordström black holes whose ADM masses differ by $\Delta M$ and whose charges differ by $\Delta Q$ will be, to leading order in $\Delta M$ and $\Delta Q$,

$$
\Delta M \approx \frac{1}{2 \pi} \frac{r_{H}-M}{r_{H}^{2}} \Delta\left(\frac{A}{4}\right)+\frac{Q}{r_{H}} \Delta Q .
$$

The term $\left(r_{H}-M\right) / r_{H}^{2}$ is the surface gravity of the static Reissner-Nordström black hole. This result has been extended to include rotation and stationary matter shells. ${ }^{19}$ One remarkable thing about these results is that they relate quantities measured at infinity and relating to the whole spacetime, such as the ADM mass, to properties solely related to the black hole and computed on its horizon, such as the area. A physical process version for this result can be given in terms of small perturbations of a background stationary spacetime. ${ }^{26}$ 
In the fully dynamical but still spherically symmetric case, a very simple relation is obtainable relating the change of area of the trapping horizon and the change of the Misner-Sharp quasi-local mass. This just uses the relation satisfied at the trapping horizon, $r=2 m$, and the areal-radius definition $A=4 \pi r^{2}$.

$$
\delta_{h} m=\frac{1}{2 \pi} \frac{1}{2 r} \delta_{h}\left(\frac{A}{4}\right) .
$$

Here and in what follows the derivatives $\delta_{h}$ refer to derivatives along the horizon. This is a special case of a more general result ${ }^{12}$ and is exact in spherical symmetry without needing to assume a stationary background or slow evolution. The formula gives a simple relation between the change in mass and the change in one quarter of the area, both quasi-local properties, without appeal to any asymptotically flat region. However, the term relating these two, $1 / 4 \pi r$, does not vanish for finite area, extremal solutions, such as occur in Reissner-Nordström spacetimes. A modification due to Hayward ${ }^{20}$ gives

$$
\delta_{h} m=\frac{1}{2 \pi} \kappa \delta_{h}\left(\frac{A}{4}\right)+w \delta_{h} V,
$$

again involving the Misner-Sharp mass in spherical symmetry, where the second term on the right-hand side can be interpreted as a work term, with $w=T_{a b}\left(l^{a} n^{b}+\right.$ $\left.n^{a} l^{b}\right) / 2$ and $V$ is the volume on a spatially flat hypersurface, $V=4 \pi r^{3} / 3$. The relation between the change in mass and change in area, denoted $\kappa$, does now vanish in extremal situations and is given by the expression (3). The price to pay for this is that the heat supply and work terms cannot be varied independently unlike the situation in ordinary thermodynamics. In ordinary thermodynamics the temperature is an intensive parameter and independent of the extensive volume, but for black holes the temperature and entropy are both related to this volume.

By restricting to slowly evolving horizons, Booth and Fairhurst ${ }^{27}$ obtained a first law of the form

$$
\delta_{h} m \approx \frac{1}{2 \pi} \kappa_{o} \delta_{h}\left(\frac{A}{4}\right) \approx \frac{1}{8 \pi} \int_{S}\left[\sigma_{l}^{2}+G_{c d} l^{c} l^{d}\right] \varepsilon_{a b} .
$$

In this case $\kappa_{o}$ denotes a constant surface gravity which can be chosen to reduce to the isolated Killing horizon value in the static limit. The first approximation is obtained only for situations near spherical symmetry allowing the Misner-Sharp definition of mass to be used, while the second approximation holds in the general case. The right-hand side can be interpreted as a flux of matter via the Einstein equations from $T_{a b} l^{a} l^{b}$ and the $\sigma_{l}^{2}$ term can be attributed to gravitational waves. As the authors point out, ${ }^{21} \mathrm{Eq}$. (7) can be interpreted in general cases as a first law without any definition of horizon energy at all.

\subsection{Second law}

The second law is perhaps the most important of the thermodynamic/mechanics relations. For event horizons it is simply the area theorem of Hawking, supplemented 
in the case of non-Einstein gravity by a number of similar arguments. ${ }^{28,29}$ An energy condition, via the equations of motion, guarantees the increase of area and this energy condition must hold also in the future. If the energy condition is violated somewhere in the future, as it is expected to be in Hawking radiation, then the event horizon entropy can be decreasing even if the energy condition is satisfied locally. ${ }^{14}$ For trapping horizons there is a simple quasi-local relation relating the area increase and the energy condition. For a congruence of curves we have

$$
\delta_{h} \varepsilon_{a b}=\theta_{h} \varepsilon_{a b},
$$

where $\delta_{h}$ denotes the variation along the congruence and $\varepsilon_{a b}$ is the area two-form of the horizon normal space (see the notes of José Luis Jaramillo ${ }^{4}$ for further details.) This equation implies that the cross-sectional area will be increasing if the expansion, $\theta_{h}$, is positive. When $h$ represents the generators of the event horizon a proof by contradiction establishes that $\theta_{h}$ cannot be negative, and hence the area is nondecreasing. Taking the derivative of the area two-form along a null direction and using the Raychaudhuri equation we have $\mathrm{e}^{30,31}$

$$
\delta_{l} \delta_{l} \varepsilon_{a b}=\left(\kappa_{l} \theta_{l}+\frac{\theta_{l}^{2}}{2}-\sigma_{l}^{2}-G_{c d} l^{c} l^{d}\right) \varepsilon_{a b} .
$$

In the perturbative limit where $\theta_{l}^{2} \approx 0$ and the transition is between two approximately non-expanding states, this equation can be integrated to obtain the HartleHawking flux law for a black hole. An event horizon cannot make a true transition between one non-expanding state and another non-expanding state because by the Raychaudhuri equation and the energy condition, $\theta_{l}$ along a null causal horizon is always decreasing.

In the case where the horizon is not necessarily null, such as the trapping horizon case, the $\theta_{h}$ can be split into a linear combination of the two normal directions $\theta_{l}$ and $\theta_{n}$. For a future trapping horizon $\theta_{l}=0$ by definition and $\theta_{n}$ is required to be negative. Since the trapping horizon is required to satisfy $\delta_{h} \theta_{l}=0$ by definition, we have

$$
\delta_{h} \varepsilon_{a b}=\frac{\theta_{n}}{\delta_{n} \theta_{l}}\left(\sigma_{l}^{2}+G_{a b} l^{a} l^{b}\right) \varepsilon_{a b} .
$$

Again this condition will hold regardless of the Einstein equations. To convert the $G_{a b} l^{a} l^{b}$ into something involving the stress-energy tensor and the energy conditions one can make use of the Einstein equations $G_{a b}=8 \pi T_{a b}$. However, in general modified theories the Einstein equations do not hold. In addition, in a general diffeomorphism invariant theory the horizon-entropy of a stationary horizon is not necessarily equal to one quarter of the area. The horizon-entropy can be computed via integration of a surface entropy density $s_{a b}$ given by the formula ${ }^{32}$

$$
s_{a b}=-2 \pi \frac{\partial \mathscr{L}}{\partial R_{c d e f}} \hat{\varepsilon}_{c d} \hat{\varepsilon}_{e f} \varepsilon_{a b}
$$

where $\mathscr{L}$ denotes the Lagrangian density, $R_{c d e f}$ is the Riemann tensor, and $\hat{\varepsilon}_{c d}$ is the antisymmetric binormal form. In the case of ordinary Einstein gravity this just 
gives one quarter of the area two-form, but in scalar-tensor theories and $f(R)$ gravity this gives the area two-form scaled by a nontrivial positive function, $s_{a b}=s \varepsilon_{a b}$. In a dynamical setting the change of this horizon-entropy is given by

$$
\delta_{h} s_{a b}=\left(\delta_{h} s+s \theta_{h}\right) \varepsilon_{a b} .
$$

The term $\left(\delta_{h} s+s \theta_{h}\right)$ needs to be positive in order for the generalized entropy to be increasing. In explicit examples, such as when $s$ represents the Brans-Dicke scalar field, ${ }^{33}$ the value of $s=\phi$ both increases and decreases along the horizon, although always remaining positive. For a causal horizon in the Brans-Dicke case we find

$$
\delta_{l}\left(\theta_{l}+\frac{l^{a} \nabla_{a} \phi}{\phi}\right)=\kappa_{l}\left(\theta_{l}+\frac{l^{a} \nabla_{a} \phi}{\phi}\right)-\frac{\theta_{l}^{2}}{2}-\sigma_{l}^{2}-\frac{(\omega+1)}{\phi^{2}}\left(l^{a} \nabla_{a} \phi\right)^{2}-\frac{8 \pi}{\phi} T_{a b} l^{a} l^{b} .
$$

If the causal horizon settles down at late times to a static horizon with $\left(\delta_{l} \phi+\right.$ $\left.\phi \theta_{l}\right)=0$ then at previous times its value cannot ever be negative because to get from a negative vale to zero its derivative must be somewhere positive, which is forbidden by (13), provided the null energy condition holds $T_{a b} l^{a} l^{b} \geq 0$ and $l$ is scaled such that $\kappa_{l}=0$.

A similar result can be obtained for quasi-local horizons, but to do this we must introduce the following requirements ${ }^{14,34}$; let $H$ be a three-dimensional hypersurface foliated by closed spacelike two-surfaces whose null normals $l$ and $n$ satisfy

$$
\begin{aligned}
\varepsilon^{a b} \delta_{l} s_{a b} & =0, \\
\varepsilon^{a b} \delta_{n} s_{a b} & <0, \\
\delta_{n}\left(\varepsilon^{a b} \delta_{l} s_{a b}\right) & <0 .
\end{aligned}
$$

These conditions are similar to those for a future outer trapping horizon and indeed reduce to them in the case of Einstein gravity where $s_{a b}=\varepsilon_{a b} / 4$. But in general these conditions are different and do not describe a surface foliated by marginally trapped surfaces. For the case of Brans-Dicke theory the first condition corresponds to $\theta_{l} \phi+\delta_{l} \phi=0$. Where $\delta_{l} \phi \neq 0$ this is not the apparent horizon. The change of the entropy is now

$$
\delta_{h} s_{a b}=\frac{\varepsilon^{c d} \delta_{n} s_{c d}}{\delta_{n}\left(\varepsilon^{e f} \delta_{l} s_{e f}\right)}\left(\frac{\theta_{l}^{2}}{2}+\sigma_{l}^{2}+\frac{\omega+1}{\phi^{2}}\left(\delta_{l} \phi\right)^{2}+\frac{8 \pi}{\phi} T_{g h} l^{g} l^{h}\right) \phi \varepsilon_{a b} .
$$

From this we see that the horizon-entropy is increasing for a horizon satisfying (14) and matter obeying the null energy condition. A horizon-entropy second law holds for these quasi-local horizons in modified gravity theories, even though they are not foliated by marginally trapped surfaces.

From the very beginning ${ }^{18}$ the association of entropy to black holes was meant as a measure of ignorance about the internal state of the black hole. As such, this interpretation of the entropy is most naturally associated with causal horizons as they are true boundaries to causal information flow. Timelike quasi-local horizons 
are certainly no boundary to two-way information flow. If instead entropy is interpreted geometrically as in Ref. 32 then an association with the conditions (14) seems natural as an entropic trapping horizon.

\section{The Conjectured Physical Properties}

The third celebrated result in black hole physics is the Hawking radiation. ${ }^{35}$ Although the Hawking result is backed by a wide range of different calculations, the result is still the subject of some controversy and alternative interpretations. Current technologies seem a long way from observing this effect for astrophysical black holes.

The prediction of Hawking radiation led to the possibility that black holes could lose mass and eventually disappear. This in turn led to the black hole information paradox $^{36}$ which in turn spawned a wide number of possible resolutions from modifying quantum mechanics to allowing information to fall into baby universes.

\subsection{Hawking radiation}

The original derivation of Hawking radiation relied on the use of null rays that approach the event horizon but still escape to infinity. ${ }^{35}$ A novel way to view the Hawking process was through the tunneling argument. ${ }^{37}$ This tunneling argument lends itself rather naturally to application on trapping horizons ${ }^{38,39}$ and recent work has focused on the use of Kodama vector fields to understand spherically symmetric situations. ${ }^{40}$ But there appear to be problems with this approach. As a very simple example, consider the following metric

$$
d s^{2}=-d t^{2}+\frac{d r^{2}}{\triangle^{2}}+\frac{r^{2} d \Omega^{2}}{\triangle}
$$

with $\triangle=1-2 M / r$. This metric is just a conformal transformation of the familiar Schwarzschild metric. It has exactly the same local null cone structure and future directed light rays propagate from near $r=2 M$ to infinity. However, the metric also has a $\theta_{l}=0$ trapping horizon at $r=3 M$ even though light rays are able to cross this surface from inside to outside without impedance. The metric is static with a timelike Killing vector field and since it is also spherically symmetric it admits a Kodama vector field that is parallel to the Killing vector field. Although the norm of the Kodama vector is tending to zero as one approaches $r=3 \mathrm{M}$, the Kodama vector field itself vanishes at $r=3 M$ which strongly suggests that using the Kodama vector field to probe the structure of this metric is likely to run into problems.

In the context of "veiled" general relativity ${ }^{41}$ this metric can even be viewed as physically equivalent to the Schwarzschild metric, with the same properties of perihelion precession, Shapiro time delay and gravitational redshift. It is therefore somewhat disconcerting that the trapping horizon and Kodama vector techniques are unable to recover the expected behavior at $r=2 M$. 
It has recently been argued that no horizon at all is necessary for Hawking radiation as long as one has the necessary structure at infinity 42,43 and that "it 'looks like' a horizon might form in the not too distant future". This occurs because of a key adiabatic approximation used in deriving the Hawking effect that the spacetime should be changing slowly with respect to the peak frequency of the radiation and allows the possibility that a spacetime may get very close to forming a horizon without one ever actually forming. It is also argued that Hawking-like radiation will occur in fuzzball geometries ${ }^{44}$ where no horizon is formed at all due to long-range quantum gravity effects.

An analogue of Hawking radiation may have been observed in the laboratory as a classical stimulated process involving analogue white hole horizons in water surface waves. ${ }^{45}$ This is a classical stimulated process rather than a quantum spontaneous process with long surface waves being converted into short deep water waves. In this case the location of the horizon is frequency dependent and is thus smeared out for a range of frequencies. The water flow that is creating the analogue geometry can be modified or switched off externally and hence there is no true event horizon. Nonetheless, a good fit to a Boltzmann factor distribution for the frequencies of the emitted waves is observed. This opens up the possibility of testing experimentally key assumptions that go into the Hawking result.

\subsection{Cosmic censorship}

The cosmic censorship conjecture ${ }^{46,47}$ is the idea that singularities of the gravitational field should not be visible to observers. The weak version forbids the existence of inextendible past directed null curves from future null infinity. In other words if a singularity forms it is covered by an event horizon and the spacetime is strongly asymptotically predictable. This builds on the Penrose singularity theorem which gives sufficient grounds for the formation of a singularity in the presence of trapped surfaces. In this way there is a link between trapped surfaces and the formation of event horizons. The cosmic censorship conjecture is very handy in classical gravity, especially without knowledge of how quantum gravity should behave, in that it allows one to define Cauchy surfaces and initial data surfaces for controlled evolution. The conjecture has been refined over the years and while there is much evidence supporting it in various guises, ${ }^{48}$ there are also known exceptions to other formulations. For example, weak cosmic censorship is violated for slow Vaidya collapse, ${ }^{49}$ although such solutions are not asymptotically flat. Weak cosmic censorship is also violated for asymptotically-flat finely-tuned self-similar collapse. ${ }^{50}$

\subsection{Penrose conjecture}

The Penrose conjecture ${ }^{51}$ states that the areal-mass of black holes cannot be greater than the total mass of a given spacetime:

$$
M \geq \sqrt{\frac{A}{16 \pi}} .
$$


This is related to and inspired by cosmic censorship. A counter-example to the Penrose conjecture is likely to involve a violation of cosmic censorship. In order to be logically independent of cosmic censorship and independent of the future evolution of the spacetime, the conjecture is usually formulated in terms of marginally trapped surfaces or at least the infimum of surfaces enclosing a marginally trapped surface. Assuming the energy conditions and Einstein equations, the minimum area needed to enclose the apparent horizon is always less than the area of the event horizon. However, it was noticed ${ }^{52}$ that the area of the trapping horizon can be greater than the area of the event horizon even though the event horizon fully encloses the trapping horizon. The Penrose inequality does not hold for the outermost apparent horizon. ${ }^{15}$

The Penrose conjecture is also actually a form of positive mass relation although it can be expressed in purely geometric terms. A related condition has been proved for time-symmetric zero extrinsic curvature initial data hypersurfaces, ${ }^{53}$ based on a idea by Geroch ${ }^{54}$ where a suitable surface is expanded outward at a rate inversely proportional to the surface's mean curvature, so-called inverse mean curvature flow. But it remains an open question as to whether the conjecture holds in general.

Related inequalities can be derived for spinning black holes in terms of their angular momentum. ${ }^{55,56}$ In particular it was proved ${ }^{56}$ that the relation

$$
A \geq 8 \pi|J|,
$$

holds for axially symmetric apparent horizons in dynamical spacetimes satisfying the dominant energy condition.

\subsection{Generalized Second Law}

The Generalized Second Law states loosely that the intrinsic entropy of horizons plus the entropy of matter outside the horizons is a non-decreasing function towards the future. The idea builds off of the area increase law for event horizons and its extensions. As we saw in the section on the second law, horizon-entropy increase results typically require an energy condition assumption. This energy condition is known to be violated in the case of Hawking radiation.

When emitting Hawking radiation the area of the black hole can actually decrease and correspondingly the horizon-entropy of the black hole decreases too. However the Hawking process produces large amounts of entropy outside of the black hole in the form of radiation and so the Generalized Second Law is expected to hold even in these cases. The Generalized Second Law can be given either as a total change

$$
\Delta S_{\text {horizon }}+\Delta S_{\text {outside }} \geq 0,
$$

in which case the change can be between a present configuration and a later equilibrium configuration, or it can be given as a more immediate differential change

$$
\frac{d}{d t}\left(S_{\text {horizon }}+S_{\text {outside }}\right) \geq 0
$$


for a suitably defined time parameter $t$ defining evolutions. The first version allows one to sidestep the issue of defining entropy in non-equilibrium situations. However, some form of non-equilibrium entropy should be defined in order to retain the Markovian property of Cauchy evolution. ${ }^{57}$ Knowledge of a possibly equilibrium past is not needed to predict the future from a present non-equilibrium state. Entropy counters such as the black hole area are certainly definable in dynamical situations. The relation between energy flux and horizon growth is more immediate for quasi-local horizons than it is for causal horizons since causal horizons can be growing, even in entirely flat space regions with no matter.

A number of proofs of the Generalized Second Law have been proposed over the years. ${ }^{58}$ Some of them can be applied equally well to causal horizons and quasi-local horizons as they depend on small perturbative deformations of isolated horizons or gedanken experiments involving transitions from one stationary state to another.

Recently it has been argued that the Generalized Second Law will only hold for null causal horizons such as the event horizon. ${ }^{59}$ In fact a proof is given there that it cannot hold in general for trapping horizons or any other kind of horizon that is not a causal horizon. The result relies on a $(1+1)$-dimensional conformal transformation of the Hartle-Hawking vacuum under the linear approximation $\theta_{l}^{2}=0$.

\subsection{Entropy bounds}

Entropy bounds are the idea that regions of spacetime can only contain a maximum amount of entropy corresponding to the equivalent entropy held in a black hole, ${ }^{60}$

$$
S \leq 2 \pi E R
$$

with $E$ the energy of the system and $R$ the areal radius, or in the so-called covariant formulation of Bousso ${ }^{61}$

$$
S \leq \frac{A}{4}
$$

The validity of this bound of course depends critically on what is meant by $S, E$ and $R .{ }^{62}$ Although this idea is not directly related to the issue of horizon definitions, similar definitional problems occur in interpreting the terms in thermodynamic relations for black holes. The bound is set by a Schwarzschild black hole for which both event horizon and apparent horizon coincide. However, it is explicitly motivated by the assumption that black holes have entropy and this entropy is related to their area. Therefore it can be seen as a proposed law of nature deriving from the association of entropy to black hole horizons. Similar, although weaker bounds, exist for uncollapsed objects. ${ }^{63}$

\subsection{Holographic principle}

The holographic principle is the conjecture that physical properties of regions of spacetime can be entirely read off from just their boundary properties. The holographic principle is in part motivated by considerations that the microstates of a 
black hole are encoded in its horizon. One can even argue that the origin of black hole entropy must be sought in the holographic principle. ${ }^{64}$ The most completely understood example of the holographic principle and somewhat independent of the black hole motivation is the AdS-CFT duality in its particular manifestation of type IIB string theory on a ten-dimensional background that is asymptotically $\mathrm{AdS}_{5} \times \mathrm{S}_{5}$ and an $N=4$ supersymmetric Yang-Mills conformal field theory that lives on the four-dimensional boundary of the $\mathrm{AdS}_{5}$.

In a more general setting, the gauge-gravity duality relates the plasma phase of strongly interacting non-Abelian gauge theories, similar to the quark-gluon phase seen at RHIC and LHC, to gravitational systems. For example, transport properties of the plasma can be studied by looking at quasi-normal models of the corresponding black holes. A related project, fluid-gravity duality, ${ }^{65}$ relates non-linear hydrodynamics and solutions of the Navier-Stokes equation to long wavelength perturbations of higher-dimensional black holes or black branes.

The fluid-gravity correspondence has been used recently ${ }^{64}$ to relate information about the behavior of the black hole to entropy currents defined for the fluid. In this case the acausal nature of the event horizon is a definite problem. In static situations it has been argued that the association of thermodynamic properties of the dual quantum field theories with the apparent horizon is more robust than with the event horizon. ${ }^{66}$

Another aspect of the fluid-gravity correspondence is the possibility of associating fluid properties to the black hole horizon itself. This is epitomized by the membrane paradigm ${ }^{67}$ in which the horizon of the black hole is replaced by a timelike stretched horizon just outside the black hole horizon which can be thought of as a surface containing all the relevant physical properties to compute electromagnetic and quantum effects in the exterior region. In early applications ${ }^{67}$ the stretched horizon was located just outside the absolute event horizon. Using the Einstein equations one can derive evolution equations for the event horizon as a viscous fluid by a two-dimensional Navier-Stokes-like equation. More recent work ${ }^{30,31,68}$ has extended this to quasi-local horizons too. One interesting result of this extension is that the bulk viscosity becomes positive for quasi-local horizons, rather than negative as with event horizons, allowing a fully "physical" evolution of the fluid equations.

\subsection{Black hole complementarity}

Black hole complementarity ${ }^{69}$ is the idea that states falling in to black holes are duplicated (somehow) on the horizon. This means that a full copy of whatever falls in to the black hole is preserved outside, while allowing for "nothing special" to be noticed by observers falling into the hole. In this way the model resolves the black hole information paradox by keeping an entire account of all states outside of the horizon.

As the focus here is on the loss of information and the irretrievable loss of this information, this argument is most naturally associated to the global event horizon 
(this is stated explicitly in Ref. 69), or rather the stretched horizon, which is somewhat loosely defined as a timelike surface a small distance outside the global event horizon, such that its area is a small value greater than the area of the global event horizon when compared along a past directed null rays (in four dimensions there are many such past null rays normal to the event horizon but because the authors are working in two dimensions which past directed null rays is not specificed).

A key point of the black hole complementarity proposal is that the duplication of information at the horizon cannot be observed by any single observer. The event horizon plays a key role in the argument because this is where the duplication is meant to take place. The teleological nature of the event horizon means this duplication process must also be teleological. A number of studies have attempted to show that there are black hole configurations for which the duplication can be observed by a single observer. ${ }^{70,71}$ For black hole complementarity to be effective these configurations cannot occur in nature. On the other hand black hole complementarity may just be wishful thinking, an attempt to solve a problem that nature solves in a completely different way, if at all.

\section{Summary}

To what extent are these properties physical, in the sense that they can be observed and tested by physicists with finite measuring apparatus? Since Hawking radiation can be detected by local observers carrying particle detectors it is perhaps the most physically measureable indication of a black hole. Hawking radiation may have a quasi-local origin, as suggested by Hájíček ${ }^{6}$ and suggested by the local nature of quantum field theory. As the example of veiled Schwarzschild shows, the existence of a trapping horizon may not even be sufficient for the production of Hawking radiation. The fact that null expansions are conformal frame dependent is one fact mitigating against trapping horizons having a physical role. If physical properties are to be conformal frame independent ${ }^{41}$ then equations like $\theta_{l}=0$ cannot qualify by themselves. One possible way to maintain the quasi-local nature but dispense with null expansions is given by the conditions (14).

In principle it should be possible to measure the temperature of the Hawking radiation of a black hole, either from a true gravitational black hole or an analogue model. This means the disagreement between the Killing-defined temperature (1) and the Kodama-defined temperature (3) is in principle decidable by experiment. Whether the non-uniqueness of the trapping horizon has any role to play in the calculation of Hawking radiation remains an open question. The relationship between the energy flux and horizon area change might also be measurable with sufficient experimental control or possibly on a purely formal level using the dual fluid-gravity correspondence. This also opens the door to the possibility that the non-uniqueness of the trapping horizon may have a counterpart in the dual fluid sector. ${ }^{64}$

The event horizon has a definite role in black hole complementarity. An event horizon in an otherwise locally flat region of spacetime must be able to duplicate 
infalling information, keeping some of it on the outside of the black hole. That this may involve highly nonlocal physics makes it hard to evaluate with current quantum field theoretic tools. The complementarity conjecture is by design impossible to verify by a single observer, unless certain regular black holes are constructable. ${ }^{70}$ The causal event horizon also has some mathematical role to play in the Generalized Second Law as its teleological nature may be essential to satisfying this rule when applied over a sufficient length of time. The very direct relationship between the behavior of a quasi-local horizon and matter fields means that the Generalized Second Law applied to trapping horizons may fail for quantum fields.

There remains much work to be done in understanding these issues. The literature currently contains a number of contradictory statements. The use of the AdS-CFT correspondence led Booth et al. ${ }^{64}$ to state that

"[The result $\left.{ }^{66}\right]$ strongly suggests that the causal boundary of a black hole is not the relevant entropy carrier..."

and consideration of the $(1+1)$-dimensional conformally coupled matter sector led Wall ${ }^{59}$ to conclude

"...the causal horizon is the only sort of horizon for which the semiclassical [Generalized Second Law] can hold."

Somewhat less spectacularly Barcelo et $a l .{ }^{42}$ demonstrate that

"...any collapsing compact object (regardless of whether or not any type of horizon ever forms) will, provided the exponential approximation and adiabatic condition hold, emit a slowly evolving Planckian flux of quanta."

whereas Hayward et al. ${ }^{40}$ state that

"[the] method [Hamilton-Jacobi tunneling for deriving Hawking radiation] works precisely for future outer trapping horizons".

To make many of the ideas we have seen above compatible may require a weakening of our current interpretations of black hole physics. The first law surface gravity relating the change in energy to the change in entropy may not be the same as the temperature of Hawking radiation. The entropy of ignorance may be carried by causal horizons while a geometric or holographic entropy is carried by quasi-local horizons. The necessary conditions for a Hawking flux of energy may not refer to any of these horizons. Further work is needed to settle which of these statements is correct, if any. Full calculations in curved space quantum field theory or quantum gravity would of course be definitive. But these theories are still incomplete and untested. The hope that we can use the physical properties of black holes to motivate ideas in quantum gravity seems problematic when there is still so much uncertainty about what it means to be a black hole. 


\section{Acknowledgments}

The author is very grateful for support from the Alexander von Humboldt Foundation and hospitality at the Max Planck Institute for Gravitational Physics in Potsdam-Golm. The author would also like to thank the organizers of the 2011 APCTP-NCTS school on gravitation.

\section{References}

1. M. Begelman and R. Rees Black Holes in the Universe (Cambridge University Press, 2010).

2. J. L. Feng and A. D. Shapere, Phys. Rev. Lett. 88 (2002) 021303 [hep-ph/0109106].

3. S. B. Giddings and S. D. Thomas, Phys. Rev. D 65 (2002) 056010 [hep-ph/0106219].

4. J. L. Jaramillo, Int. J. Mod. Phys. D 20 (2011) 2169.

5. J. M. M. Senovilla, Int. J. Mod. Phys. D 20 (2011) 2139.

6. P. Hajicek, Phys. Rev. D 36 (1987) 1065.

7. W. A. Hiscock, Phys. Rev. D 40 (1989) 1336.

8. W. Collins, Phys. Rev. D 45 (1992) 495.

9. S. A. Hayward, Phys. Rev. D 49 (1994) 6467.

10. A. Ashtekar, J. Baez, A. Corichi and K. Krasnov, Phys. Rev. Lett. 80 (1998) 904.

11. M. Visser, Int. J. Mod. Phys. D 12 (2003) 649.

12. A. Ashtekar and B. Krishnan, Living Rev. Rel. 7 (2004) 10.

13. A. B. Nielsen, M. Jasiulek, B. Krishnan and E. Schnetter, Phys. Rev. D 83 (2011) 124022 .

14. A. B. Nielsen, Class. Quant. Grav. 27 (2010) 245016.

15. I. Ben-Dov, Phys. Rev. D 70 (2004) 124031.

16. R. Penrose, Phys. Rev. Lett. 14 (1965) 57.

17. S. W. Hawking, Phys. Rev. Lett. 26 (1971) 1344.

18. J. D. Bekenstein, Phys. Rev. D 7 (1973) 2333.

19. J. M. Bardeen, B. Carter and S. W. Hawking, Commun. Math. Phys. 31 (1973) 161.

20. S. A. Hayward, Class. Quant. Grav. 15 (1998) 3147.

21. I. Booth and S. Fairhurst, Phys. Rev. D 75 (2007) 084019.

22. A. B. Nielsen, Gen. Rel. Grav. 41 (2009) 1539.

23. A. B. Nielsen and J. H. Yoon, Class. Quant. Grav. 25 (2008) 085010.

24. M. Pielahn, G. Kunstatter and A. B. Nielsen, arXiv:1103.0750 [gr-qc].

25. A. B. Nielsen, Phys. Rev. D 74 (2006) 044038 [gr-qc/0603127].

26. R. M. Wald, Quantum Field Theory in Curved Spacetimes and Black Hole Thermodynamics (University of Chicago Press, Chicago, 1994).

27. I. Booth and S. Fairhurst, Phys. Rev. Lett. 92 (2004) 011102.

28. T. Jacobson, G. Kang and R. C. Myers, Phys. Rev. D 52 (1995) 3518.

29. G. Kang, Phys. Rev. D 54 (1996) 7483.

30. E. Gourgoulhon and J. L. Jaramillo, Phys. Rev. D 74 (2006) 087502.

31. E. Gourgoulhon and J. L. Jaramillo, New Astron. Rev. 51 (2008) 791.

32. R. M. Wald, Phys. Rev. D 48 (1993) 3427.

33. M. A. Scheel, S. L. Shapiro and S. A. Teukolsky, Phys. Rev. D 51 (1995) 4236.

34. V. Faraoni and A. B. Nielsen, arXiv:1103.2089 [gr-qc].

35. S. W. Hawking, Commun. Math. Phys. 43 (1975) 199.

36. J. Preskill, [hep-th/9209058].

37. M. K. Parikh and F. Wilczek, Phys. Rev. Lett. 85 (2000) 5042. 
38. R. Di Criscienzo, M. Nadalini, L. Vanzo, S. Zerbini and G. Zoccatelli, Phys. Lett. B657 (2007) 107 [arXiv:0707.4425 [hep-th]].

39. A. B. Nielsen and D. H. Yeom, Int. J. Mod. Phys. A 24 (2009) 5261.

40. S. A. Hayward, R. Di Criscienzo, L. Vanzo, M. Nadalini and S. Zerbini, Class. Quant. Grav. 26 (2009) 062001.

41. N. Deruelle and M. Sasaki, arXiv:1007.3563 [gr-qc].

42. C. Barcelo, S. Liberati, S. Sonego and M. Visser, Phys. Rev. D 83 (2011) 041501.

43. C. Barcelo, S. Liberati, S. Sonego and M. Visser, J. High. Energy Phys. 1102 (2011) 003.

44. B. D. Chowdhury and S. D. Mathur, Class. Quant. Grav. 26 (2009) 035006.

45. S. Weinfurtner et al., Phys. Rev. Lett. 106 (2011) 021302.

46. R. M. Wald, Gravitational collapse and cosmic censorship, in Black Holes, Gravitational Radiation and the Universe: Essarys in Honor of C.V. Vishveshwara, eds. B. R. Iyer and B. Bhawal (Kluwer Academic, Dordrecht, 1999) [gr-qc/9710068].

47. H. Ringstrom, Living Rev. Rel. 13 (2010) 2.

48. S. Hod, Phys. Rev. Lett. 100 (2008) 121101.

49. W. A. Hiscock, L. G. Williams and D. M. Eardley, Phys. Rev. D 26 (1982) 751.

50. M. W. Choptuik, Phys. Rev. Lett. 70 (1993) 9.

51. M. Mars, Class. Quant. Grav. 26 (2009) 193001.

52. P. S. Jang and R. M. Wald, J. Math. Phys. 18 (1977) 41.

53. G. Huisken and T. Ilmanen, J. Diff. Geom. 59 (2001) 353.

54. R. Geroch, Annals N. Y. Acad. Sci. 224 (1973) 108.

55. I. Booth and S. Fairhurst, Phys. Rev. D 77 (2008) 084005.

56. J. L. Jaramillo, M. Reiris and S. Dain, [arXiv:1106.3743 [gr-qc]].

57. A. Corichi, D. Sudarsky, Mod. Phys. Lett. A 17 (2002) 1431.

58. A. C. Wall, J. High Energy Phys. 0906 (2009) 021.

59. A. C. Wall, [arXiv:1105.3520 [gr-qc]].

60. J. D. Bekenstein, Phys. Rev. D 23 (1981) 287.

61. R. Bousso, J. High Energy Phys. 9907 (1999) 004.

62. D. N. Page, J. High Energy Phys. 0810 (2008) 007.

63. G. Abreu and M. Visser, J. High Energy Phys. 1103 (2011) 056.

64. I. Booth, M. P. Heller, G. Plewa and M. Spalinski, Phys. Rev. D 83 (2011) 106005.

65. S. Bhattacharyya, V. E. Hubeny, S. Minwalla and M. Rangamani, J. High Energy Phys. 0802 (2008) 045.

66. P. Figueras, V. E. Hubeny, M. Rangamani and S. F. Ross, J. High Energy Phys. 0904 (2009) 137.

67. R. H. Price and K. S. Thorne, Phys. Rev. D 33 (1986) 915.

68. E. Gourgoulhon, Phys. Rev. D 72 (2005) 104007.

69. L. Susskind, L. Thorlacius and J. Uglum, Phys. Rev. D 48 (1993) 3743.

70. D.-H. Yeom and H. Zoe, Phys. Rev. D 78 (2008) 104008.

71. S. E. Hong, D.-I. Hwang, D.-H. Yeom and H. Zoe, J. High Energy Phys. 0812 (2008) 080 . 